ПСИХОЛОГИЧЕСКИЕ ФАКТОРЫ В СНИЖЕНИИ ИЗБЫТОЧНОЙ МАССЫ ТЕЛА ПОСЛЕ БАРИАТРИЧЕСКИХ ОПЕРАЦИЙ

\author{
(c) А.Е. Неймарк ${ }^{1 *}$, Ш.А. Еганян ${ }^{1}$, М.И. Гальченко²
}

1 ФГБУ «Национальный медицинский исследовательский центр им. В.А. Алмазова» Минздрава России, Санкт-Петербург, Россия;

${ }^{2}$ ФГБОУ ВО «Санкт-Петербургский государственный аграрный университет», Санкт-Петербург, Россия

Обоснование. Актуальность проблемы ожирения в настоящее время не вызывает сомнений. Одним из наиболее эффективных методов лечения сегодня является бариатрическая хирургия. В то же время результативность хирургического метода не всегда прогнозируема, так как подвержена влиянию различных факторов, в том числе психологическому состоянию пациента.

Цель. Изучить психологические особенности пациентов с ожирением до и после хирургического (бариатрического) лечения и установить взаимосвязи со снижением веса.

Методы. В исследовании приняли участие 32 человека с морбидным ожирением до и через год после лапароскопической продольной резекции желудка с исходным индексом массы тела 45,36 кг/м² (+54,90;-33,00). Использовался опросник: Symptom Check List-90-R (L.R. Derogatis).

Результаты. Наличие или повышение уровня фобической тревожности (стойкая реакция страха на определенных людей, ситуации и места) и психотизма (замкнутость, межличностная изоляция, избегание) у пациента может оказывать негативное воздействие на успешность снижения веса в послеоперационный период.

Заключение. Выявлены психологические факторы, влияющие на прогноз снижения массы тела после выполненной бариатрической операции. Психологическое тестирование и определение психотипа пациента может иметь прогностическую ценность для улучшения результатов хирургического лечения ожирения.

КЛЮЧЕВЫЕ СЛОВА: морбидное ожирение; бариатрическая хирургия; психологические факторы; эффективность бариатрии; мультидисциплинарный подход.

\title{
PSYCHOLOGICAL FACTORS IN REDUCING EXCESS BODY WEIGHT AFTER BARIATRIC SURGERY
}

(c) Aleksandr E. Neimark*, Shushanna A. Eganian', Maxim I. Galchenko²

${ }^{1}$ «Almazov National Medical Research Centre» of the Ministry of Health of the Russian Federation, St. Petersburg, Russia; ${ }^{2}$ Saint-Petersburg State Agrarian University, St. Petersburg, Russia

BACKGROUND: The relevance of the problem of obesity today is not in doubt. One of the most effective treatments today is bariatric surgery. At the same time, the effectiveness of the surgical method is not always predictable, as it is influenced by various factors, including the psychological state of the patient.

AIM: To study the psychological characteristics of patients with obesity before and after surgical (bariatric) treatment and to establish the relationship with weight loss.

MATERIALS AND METHOD: The study involved 32 patients with morbid obesity before and a year after laparoscopic longitudinal resection of the stomach with an initial body mass index of $45.36(+54.90 ;-33.00)$. Questionnaire used: Symptom Check List-90-R (L.R. Derogatis). Statistical data processing was carried out using the statistical programming language R.

RESULTS: The presence or increase in the level of phobic anxiety (a persistent reaction of fear to certain people, situations and places) and psychotism (isolation, interpersonal isolation, avoidance) in a patient can have a negative impact on the success of weight loss in the postoperative period.

CONCLUSIONS. The psychological factors affecting the prognosis of weight loss after bariatric surgery have been identified. Psychological testing and determining the patient's psychotype can be predictive in improving the outcome of surgical treatment of obesity.

KEYWORDS: morbid obesity; bariatric surgery; psychological factors; bariatric efficacy; multidisciplinary approach.

\section{ОБОСНОВАНИЕ}

Сегодня, несмотря на активную пропаганду здорового образа жизни и питания, большинство стран охвачены «эпидемией» ожирения. Количество пациентов, страдающих ожирением, превышает 650 млн человек. Актуальность проблемы ожирения заключается еще и в том, что количество людей с избыточным весом прогрессивно увеличивается. Морбидное ожирение, при котором индекс массы тела более 40 кг/м², является при- 
чиной развития серьезных заболеваний сердечно-сосудистой и опорно-двигательной систем, сахарного диабета 2 типа, что, в свою очередь, снижает продолжительность и качество жизни человека [1-4].

Для большинства пациентов, страдающих морбидным ожирением, лечение с помощью консервативных методов (диетотерапия, фармакотерапия, физические нагрузки) малоэффективно. Одним из наиболее эффективных способов лечения ожирения стала бариатрическая хирургия. Хирургическое лечение позволяет не только снизить вес, но и существенно сократить частоту развития сопутствующих ожирению заболеваний (артериальная гипертензия, сахарный диабет 2 типа, ишемическая болезнь сердца), тем самым увеличивая продолжительность жизни человека и улучшая ее качество [2]. Несмотря на значимую эффективность, хирургическое лечение - лишь одна из составляющих комплексного подхода к лечению ожирения, поскольку его результативность значительно подвержена влиянию различных факторов, в том числе психологического состояния пациента. В связи с этим актуальной является работа мультидисциплинарной команды (эндокринолог, психолог, диетолог и др.) как до, так и после бариатрической операции $[4,5]$.

Появляются научные исследования, в которых проводится комплексная оценка состояния пациентов с морбидным ожирением до и после выполнения бариатрических операций $[3,6,7,8]$. При этом остаются малоизученными психологические факторы, участвующие в снижении избыточной массы тела в послеоперационный период, выявление которых может иметь прогностическую ценность для улучшения результатов лечения морбидного ожирения [9]. В связи с этим одним из важных компонентов комплексного лечения является проведение психологической диагностики состояния пациентов и психокоррекционных мероприятий в периоды до и после проведения бариатрических операций. Важно отметить, что психологическое сопровождение пациента является неотъемлемой составляющей хирургического лечения ожирения $[2,4,6,8]$.

\section{ЦЕЛЬ}

Изучить психологические особенности пациентов с ожирением до и после бариатрической операции и установить взаимосвязи со снижением веса.

\section{МЕТОДЫ}

\section{Дизайн исследования}

Исследование одноцентровое проспективное неконтролируемое неослепленное. В исследование включены 32 человека сморбидным ожирениемдо ичерез годпосле лапароскопической продольной резекции желудка с исходным индексом массы тела 45,36 кг/м² (+54,90;-33,00). Использовался опросник: Symptom Check List-90-R (L.R. Derogatis).

\section{Критерии соответствия}

Критерии включения: ожирением с индексом массы тела более 40 или более 35 кг/м² при наличии сопутствующих метаболических нарушений - артериальная гипертензия, дислипидемия, сахарный диабет 2 типа.

\section{Условия проведения}

В исследовании приняли участие пациенты, проходившие хирургическое лечение морбидного ожирения на базе Национального медицинского исследовательского центра им. В.А. Алмазова Министерства здравоохранения Российской Федерации, Санкт-Петербург, Россия.

\section{Продолжительность исследования}

Исследование проводилось в период с октября 2017 г. по апрель 2019 г. Продолжительность периода включения в исследование - с момента госпитализации до 12-18 мес после хирургического вмешательства. До выполнения бариатрической операции проводилась психологическая диагностика. Анализ данных осуществлялся в послеоперационном периоде в сроки от 12 до 18 мес от момента выполненной операции, в период окончательной стабилизации снижения массы тела. В эти сроки пациент приглашался на контрольное взвешивание.

\section{Описание медицинского вмешательства}

Психологическая диагностика проводилась в индивидуальном порядке в присутствии клинического психолога. Проведена ознакомительная беседа с пациентами с пояснением целей исследования. Всем обследуемым предлагалось заполнить опросник психопатологической симптоматики, состоящий из 90 вопросов. Перед началом психодиагностики была разъяснена инструкция к ее выполнению. Пациенты приглашались на контрольное взвешивание в сроки от 12 до 18 мес от момента выполненной операции.

Основной исход исследования

Распространенность ожирения среди лиц трудоспособного возраста.

\section{Дополнительные исходы исследования}

Эффективность хирургических методов лечения ожирения.

\section{Анализ в подгруппах}

Было исследовано 32 человека с морбидным ожирением: 23 женщины и 9 мужчин. Исходный средний индекс массы тела исследуемых составляет 45,36 кг/м² (+54,90;-33,00). Средний возраст - 44 года $(+61 ;-19)$.

\section{Методы регистрации исходов}

1. Опросник выраженности психопатологической симптоматики (Symptom Check List-90-R), созданный L.R. Derogatis. Опросник состоит из 90 вопросов и содержит следующие шкалы: соматизация (SOM), обсессивность-компульсивность (О-С), межличностная сенситивность (INT), депрессия (DEP), тревожность (Anx), враждебность (Hos), фобическая тревожность (Phob), паранойяльность (Par), психотизм (Psy), общий индекс тяжести симптомов (GSY), индекс наличного симптоматического дистресса (PSDY) [10].

2. Методы статистического анализа данных.

Этическая экспертиза

Этическая экспертиза не была проведена. 


\section{Статистический анализ}

Принципы расчета размера выборки. Размер выборки предварительно не рассчитывался.

Методы статистического анализа данных.

Статистическая обработка данных осуществлялась с помощью языка статистического программирования $\mathrm{R}$ (версия 3.5, относится к категории свободного программного обеспечения, разработка координируется R Foundation, Austria) с использованием IDE RStudio. Для разделения групп применялся кластерный анализ, алгоритм Fuzzy C-Means (библиотека cluster). С учетом наличия небольших групп и отсутствия нормальности в них, применялись непараметрические методы. Для сравнения изучаемых показателей в группах использовался критерий Манна-Уитни-Уилкоксона (в случае множественных сравнений применялась корректировка Беньямини-Йекутили), критерий Краскела-Уоллиса. Для проверки унимодальности использовался dipтест Хартигана (Hartigan's dip test, библиотека diptest). В корреляционном анализе использовался коэффициент корреляции Спирмена (функция rcorr библиотеки Hmisc). Уровень значимости, применяемый в исследовании: $a=0,05$ [11].

\section{РЕЗУЛЬТАТЫ}

Объекты (участники) исследования

32 человека с морбидным ожирением: 23 женщины и 9 мужчин. Исходный средний индекс массы тела

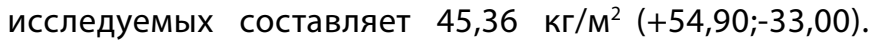
Средний возраст - 44 года (+61;-19). Всем пациентам показана лапароскопическая продольная резекция желудка в качестве хирургического метода лечения ожирения.

\section{Основные результаты исследования}

Статистическая обработка данных, полученных с помощью опросника выраженности психопатологической симптоматики «SCL-90-R», выявила отсутствие унимодальности по части показателей в группе, состоящей из 32 человек, что говорит о возможном наличии в ней нескольких подгрупп.

Таким образом, кластерный анализ позволил разделить исследуемую группу на две подгруппы: 1 подгруппа (19 человек) с очень низким уровнем выраженности психопатологической симптоматики и более благополучным текущим психологическим состоянием и 2 подгруппа (13 человек), в которой исследуемый симптоматический комплекс умеренно выражен. Различия между подгруппами практически по всем исследуемым параметрам статистически значимы на заданном уровне (за исключением шкалы «паранойяльность») (табл. 1).

При этом между подгруппами нет статистически значимых различий по потере избыточной массы тела, в обеих подгруппах отмечается успешное прогнозируемое снижение веса (1 подгруппа: \%EWL - 47\%; 95\% ДИ [44-55], 2 подгруппа: \%EWL - 50\%; 95\% ДИ [40-53\%], рис. 1, 2).

Пациенты второй подгруппы предъявляли большое количество выраженных соматических жалоб (SOM 1,5 балла; 95\% ДИ [0,92-1,67]). С одной стороны, это объяснялось проявлением симптомов сопутствующих соматических заболеваний, связанных с морбидным
Табл. 1. Результаты теста Манна-Уитни-Уилкоксона по полученным группам

\begin{tabular}{lcc}
\hline \multicolumn{1}{c}{ Показатель } & Статистика, W & p-value \\
\hline O-C & 20,5 & 0,00008 \\
INT & 42,0 & 0,002 \\
DEP & 24,0 & 0,0001 \\
Anx & 10,0 & 0,00001 \\
Hos & 24,5 & 0,0001 \\
Phob & 36,0 & 0,0003 \\
Par & 84,0 & 0,10 \\
Psy & 61,5 & 0,016 \\
GSY & 1,0 & 0,000003 \\
PST & 0,0 & 0,000002 \\
PSDY & 20,5 & 0,00007 \\
\%EWL & 118,5 & 0,86 \\
\hline
\end{tabular}

ожирением (сердечно-сосудистая патология, сахарный диабет 2 типа, нарушения опорно-двигательного аппарата). С другой стороны, это объяснялось тенденцией к физиологическим нарушениям в различных органах и системах организма, причиной возникновения которых является психическое напряжение и дистресс. Зарубежные исследования показывают, что соматические расстройства являются основными факторами, которые усиливают выраженность психопатологической симптоматики и негативно влияют на психологическое здоровье пациентов, страдающих морбидным ожирением [12].

Также пациенты данной подгруппы умеренно склонны переживать навязчивые мысли и чувства (О-С 0,90 балла; 95\% ДИ [0,7-1]). Они отмечают у себя тревожно-депрессивную симптоматику (Anx - 0,8 балла, 95\% ДИ [0,5-1,2], DEP - 0,85 балла, 95\% ДИ [0,77-1,15]) преобладание подавленного настроения, упадка сил, снижение интереса, негативной оценки себя и своего состояния; при этом у них присутствует слабовыраженная тенденция к враждебным проявлениям, негативным эмоциям по отношению к другим людям и ситуациям (Hos - 0,85 балла, 95\% ДИ [0,77-1,15]). Такая совокупность симптомов может являться причиной возникновения дистресса у пациентов с ожирением, что подтверждается высокими показателями общего индекса переживания тяжести симптомов (GSY - 0,78 балла, 95\% ДИ [0,61-0,87]).

Более подробное изучение 2 подгруппы, для которой исследуемый симптоматический комплекс умеренно выражен, выявило статистически значимую корреляционную отрицательную связь между шкалой «фобическая тревожность» и процентом снижения избыточной массы тела ( $r=-0,63 ; p=0,02)$, а также между шкалой «психотизм» и процентом снижения избыточной массы тела $(r=-0,63$; $\mathrm{p}=0,02)$. Структура связи значений по шкале психотизма 
Cluster silhouette plot

Average silhouette width: 0.59

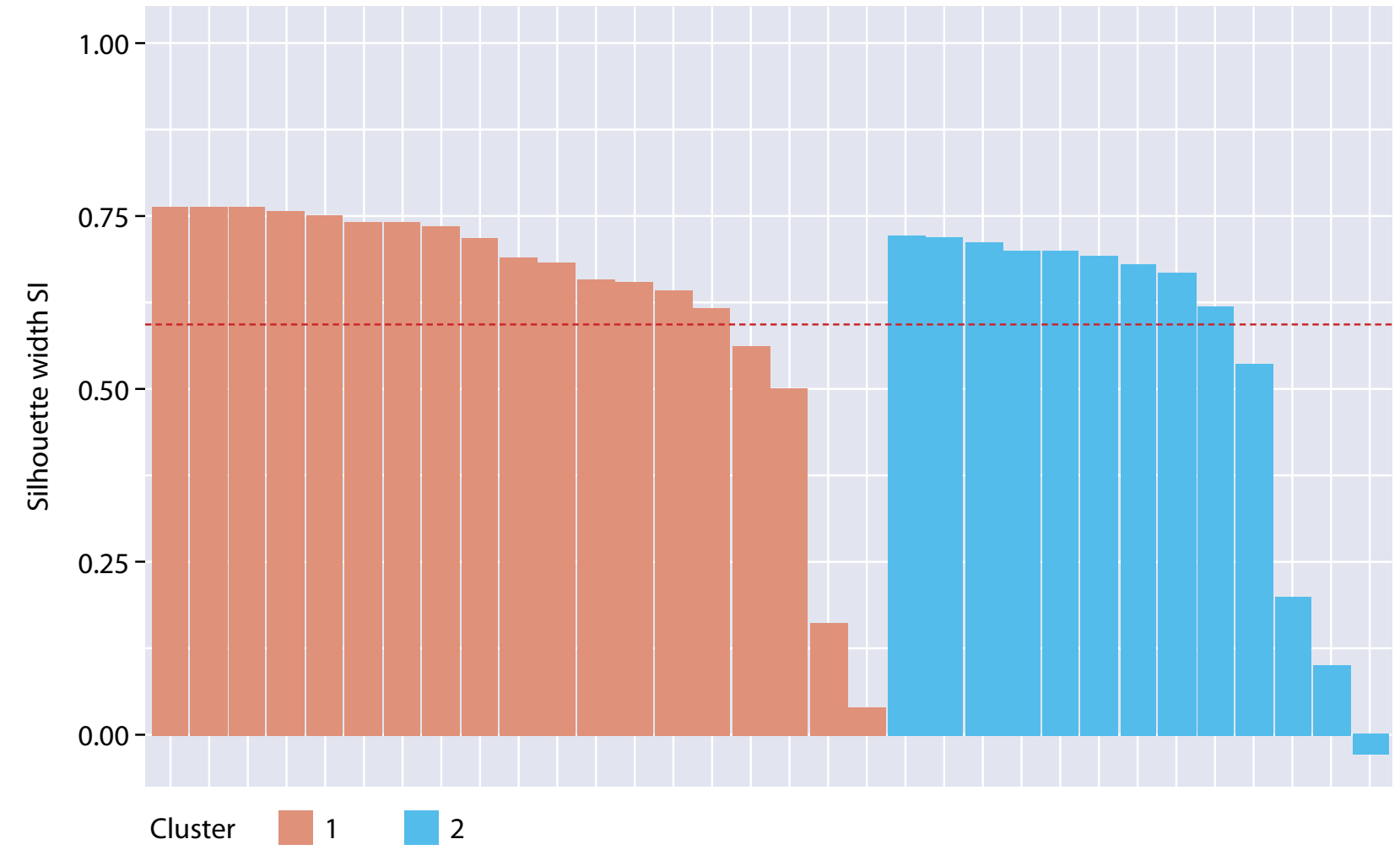

Рисунок 1. Структура силуэтов кластеров

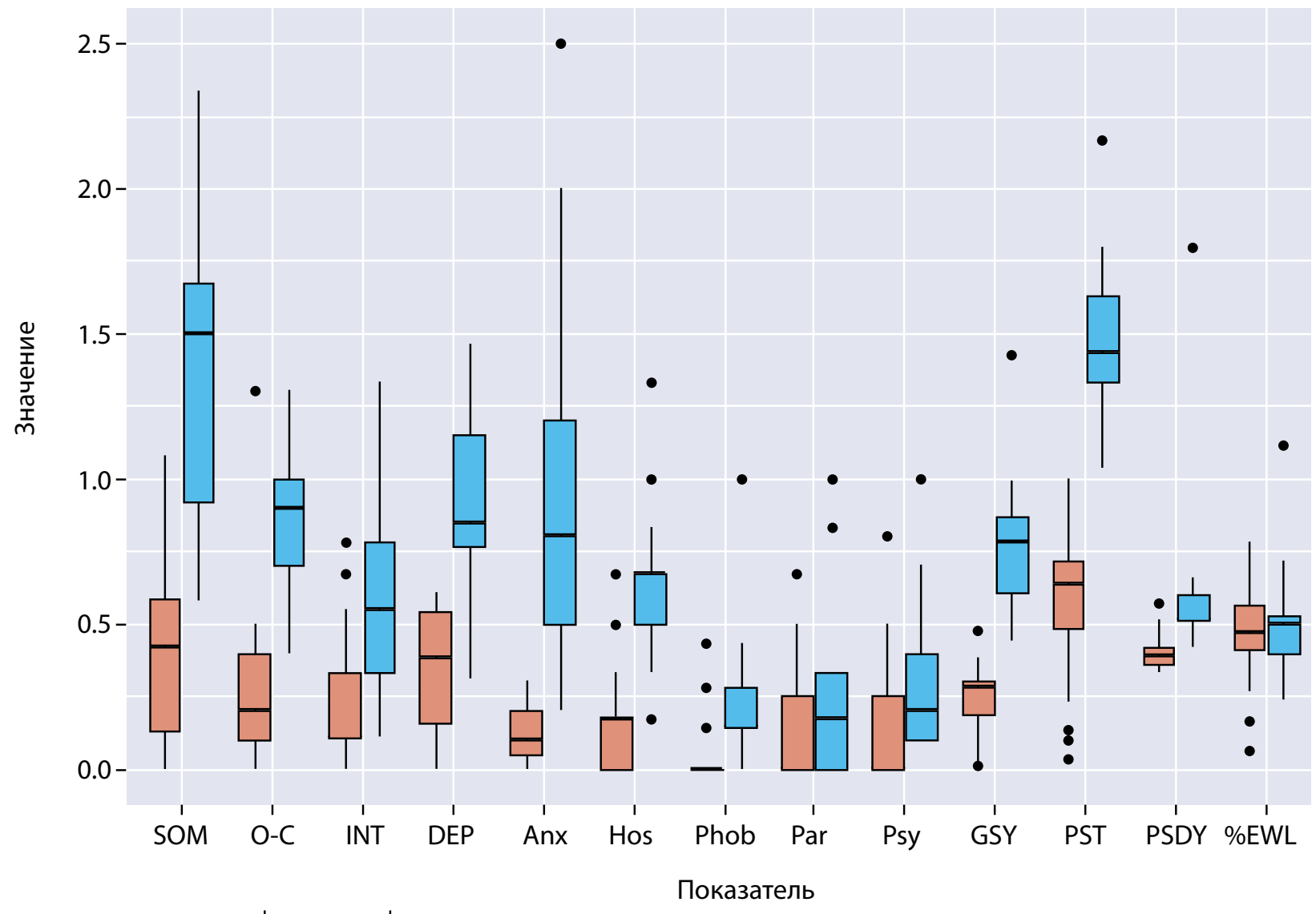

Группа Е 1 乌 2

Рисунок 2. Различия по показателям в зависимости от группы

(значения PST масштабированы делением на 30, значения PSDY масштабированы умножением на 30). 
и процента снижения массы тела во 2 группе предельно интересна (рис. 3).

Фактически коэффициент корреляции показал, что, исходя из имеющихся данных, малые значения по шкале «психотизм» (Psy) соответствуют большему проценту потери веса (\%EWL) (приблизительно от 0,5 до 1,2), начиная со значения 0,25 - около 0,3. Действительно, если использовать данное деление, то для 2 группы различия значимы (W=178,5; p-value=0,006, рис. 4).

Потеря веса у пациентов с большими 0,25 значениями по шкале «психотизм» в среднем меньше (Med=0,38, 95\% ДИ $[0,24-0,44])$, чем у пациентов с низкими значениями по данной шкале (Med=0,52; 95\% ДИ [0,50-0,72]).

Можно предположить, что наличие или повышение уровня фобической тревожности (стойкая реакция стра- ха на определенных людей, ситуации и мест) и психотизма (замкнутость, межличностная изоляция, избегание) у пациента может оказывать негативное воздействие на успешность снижения веса в послеоперационный период.

\section{Дополнительные результаты исследования}

В статье представлены все полученные в ходе исследования результаты. Данные описаны в полном объеме.

\section{Нежелательные явления}

В данном исследовании отсутствовали нежелательные явления. Специалистами была обеспечена анонимность полученных психологических данных в каждом отдельном случае.

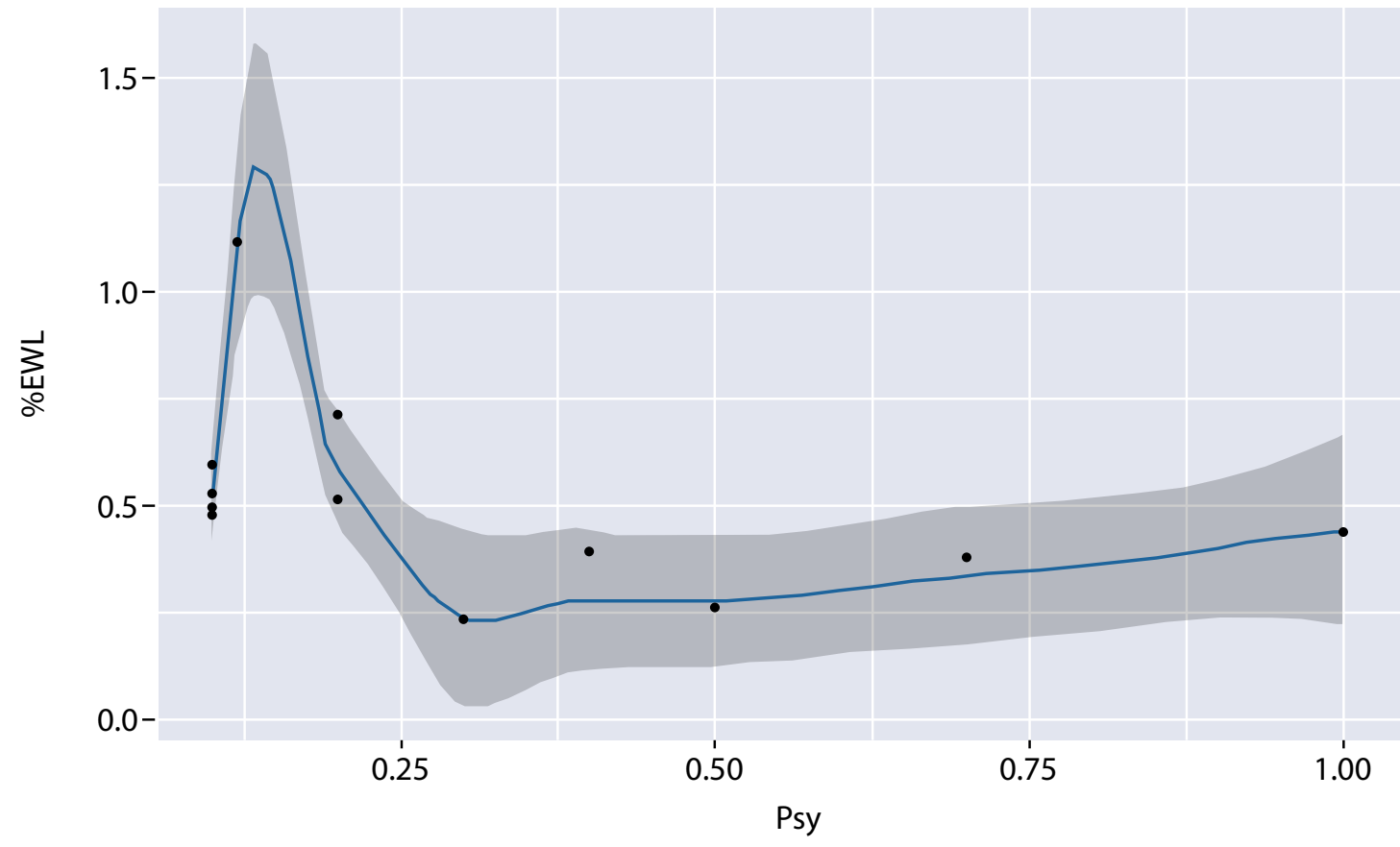

Рисунок 3. Структура связи значений по шкале «психотизм» и потери веса.

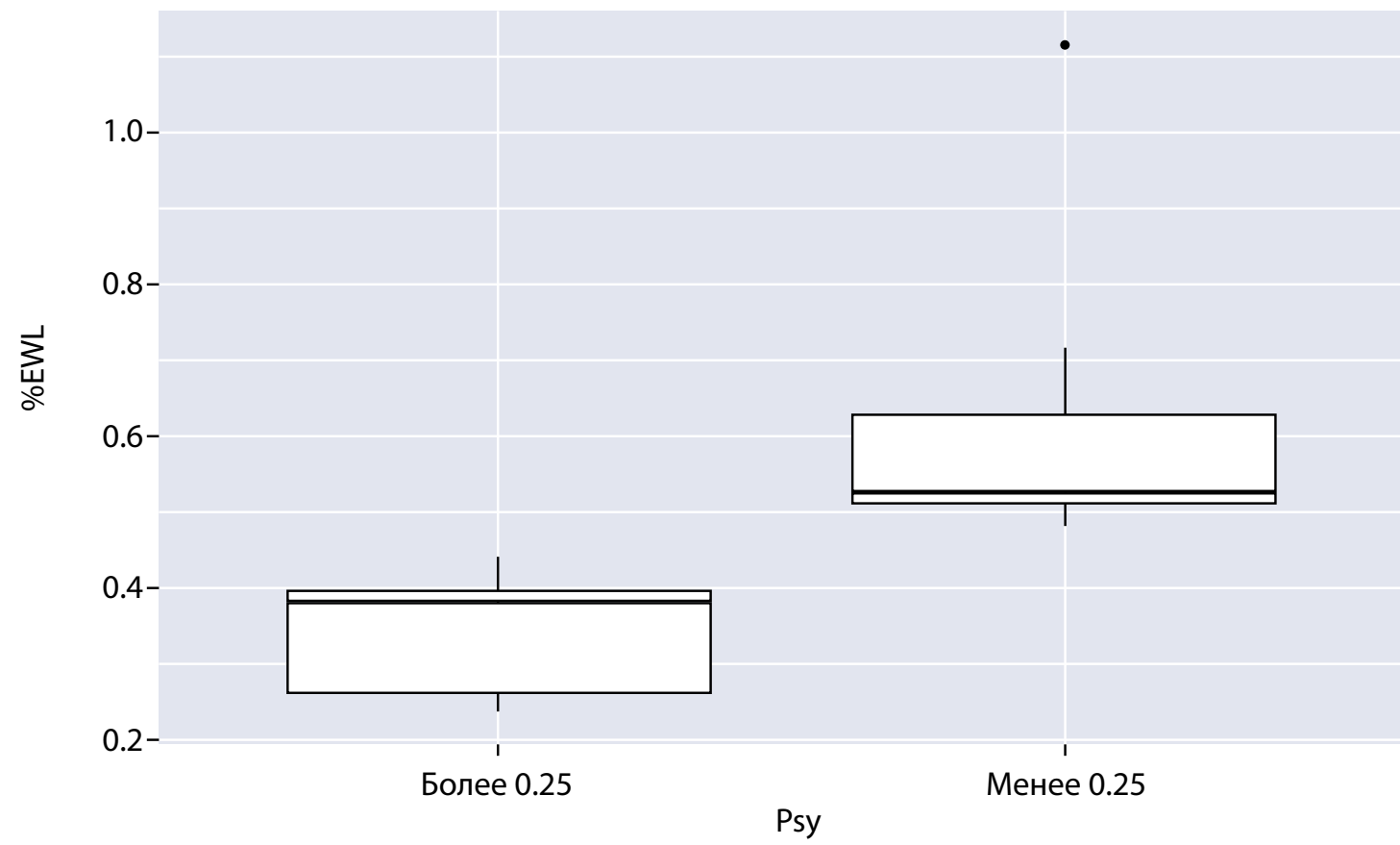

Рисунок 4. Потеря веса в зависимости от значения по шкале «психотизм». 


\section{ОБСУЖДЕНИЕ}

Резюме основного результата исследования

Исследование позволило выявить особенности психологического состояния пациентов с морбидным ожирением до начала лечения, которое у 13 из них характеризовалось сильной выраженностью соматических жалоб и переживанием дистресса, наличием тревожно-депрессивной симптоматики. Исследование показало, что у пациентов с низким уровнем «психотизма» (замкнутость, межличностная изоляция, избегание) и «фобической тревожности» (стойкая реакция страха на определенных людей, ситуации и мест) происходило более успешное снижение веса после бариатрической операции. Оценка данных показателей до хирургического вмешательства поможет спрогнозировать процент снижения массы тела у каждого пациента в послеоперационный период.

\section{Обсуждение основного результата исследования}

По результатам данного исследования выявлена подгруппа пациентов (13 человек), у которых необходимо проведение психологической коррекции, где мишенями работы должны являться такие выявленные эмоциональные расстройства, как дистресс и тревожно-депрессивная симптоматика.

Можно предположить, что снижение уровня фобической тревожности (проработка страхов) и замкнутости (социальное раскрепощение) с помощью психокоррекционной работы поможет пациенту более успешно снижать вес после бариатрической операции. Также данные показатели могут являться прогностическими критериями при оценке результатов успешности снижения веса после хирургического вмешательства.

Полученные результаты могут являться основой программы психологической коррекции для пациентов с морбидным ожирением. Такие эмоционально-личностные особенности пациентов, как «фобическая тревожность» и «психотизм», наряду с другими параметрами должны являться основными мишенями при проведении психокоррекционных мероприятий как до, так и после выполнения бариатрических операций.

В дальнейшем планируется включение в данное исследование большего количества пациентов, перенесших бариатрическую операцию с последующей разработкой программы психокоррекции эмоционально-личностных особенностей, препятствующих успеш- ному снижению избыточной массы тела в послеоперационный период.

\section{Ограничения исследования}

Ограниченность результатов исследования может быть связана с небольшой выборкой и неравномерным распределением по полу (выборка состоит из 32 человек, из них 23 женщины и 9 мужчин). Статистическая обработка данных выявила отсутствие унимодальности по части показателей в исследуемой группе, состоящей из 32 человек, что говорит о наличии в ней нескольких подгрупп, что не позволяет применить полученные результаты ко всем участникам исследования.

\section{ЗАКЛЮЧЕНИЕ}

В проведенном исследовании выявлены психологические факторы, влияющие на снижение избыточной массы тела в послеоперационный период. Ожирение, как полиэтиологическое заболевание, требует мультидисциплинарного подхода. Наличие специалиста-психолога необходимо, в том числе, и при хирургическом лечении. Психологическое тестирование и определение психотипа пациента могут иметь прогностическую ценность для улучшения результатов хирургического лечения ожирения.

\section{ДОПОЛНИТЕЛЬНАЯ ИНФОРМАЦИЯ}

Источник финансирования. Исследование выполняется при финансовой поддержке гранта РНФ №17-75-30052.

Конфликт интересов. Авторы декларируют отсутствие явных и потенциальных конфликтов интересов, связанных с публикацией настоящей статьи.

\section{Участие авторов.}

Неймарк А.Е. выдвинул основную гипотезу, концепцию и дизайн исследования, определил объект исследования, оказал содействие в организации проведения исследования.

Еганян Ш.А. организовала исследование, осуществила выбор методик и формат исследования. Провела обработку и анализ эмпирических данных, определила практическую значимость исследования.

Гальченко М.И. - под руководством и при непосредственном участии автора собраны материалы исследования и выполнена статистическая обработка данных. Все авторы внесли значимый вклад в проведение исследования и подготовку статьи, прочли и одобрили финальную версию статьи перед публикацией.

\section{СПИСОК ЛИТЕРАТУРЫ | REFERENCES}

1. Гришкян Д.Р. Стратегия и тактика диагностики и хирургического лечения ожирения и его последствий: Автореф. ... дис. д-ра мед.наук. - M., 2010. - 31c. [Grishkyan D.R. Strategy and tactics of diagnosis and surgical treatment of obesity and itsconsequences: Abstract. ... dis. Dr. med.nauk. - M., 2010. - 31c. (In Russ).].

2. Клинические рекомендации по бариатрической и метаболической хирургии / Российское общество бариатрических хирургов, М. - 2014. [Clinical recommendations for bariatric and metabolic surgery / Russian Society of Bariatric Surgeons, M. - 2014. (In Russ).].

3. Марголин М., Шмелёва Н., Марголина Е., Пликшс А., Трофимович Г. Дооперационная психологическая оценка пациента, как критерий эффективности бандажирования желудка / Материалы Пятого российского симпозиума с международным участием 25-27 июня 2009 г. / Хирургическое лечение ожирения и метаболических нарушений / под ред. Ю.И. Яшкова, М.С. Синеокой 2009. - C.47. [Margolin M., Shmelyova N., Margolina E., Pleiks A., Trofimovich G. Preoperative psychological evaluation of the patient as a criterion of the effectiveness of gastric banding / Proceedings of the Fifth Russian Symposium with International Participation June 25-27, 2009 / Surgical treatment of obesity and metabolic disorders / ed. Yu.I. Yashkov, M.S. Sineokaya - 2009. P.47. (In Russ).]. 
4. Kubik JF, Gill RS, Laffin M, Karmali S. The Impact of Bariatric Surgery on Psychological Health. J Obes. 2013;2013(4):1-5. doi: https://doi.org/10.1155/2013/837989

5. Завражных Л.А., Смирнова Е.Н., Зиньковская Т.М. Влияние психологического профиля на эффективность лечения больных метаболическим синдромом // Практическая медицина. 2010. №43. - C.118-121. [Zavrazhnykh LA, Smirnova EN, Zinkovskaya TM. Influence of the psychological profile on the effectiveness of treatment of patients with metabolic syndrome // Practical medicine. 2010; №43. - P. 118-121. (In Russ).].

6. Фирсова Л.Д., Аскерханов Р.Г. Психотерапевтическая поддержка пациентов после бариатрических операций / Сборник тезисов 5-6 марта 2015 г. 41-я научная сессия ЦНИИГ «Расширяя границы». - M. - C. 62. [Firsova LD, Askerkhanov RG Psychotherapeutic support of patients after bariatric operations / Proceedings book March 5-6, 201541 st scientific session of the CNIIG «Expanding borders». M. - P. 62. (In Russ).]

7. Dawes AJ, Maggard-Gibbons M, Maher AR, et al. Mental Health Conditions Among Patients Seeking and Undergoing Bariatric Surgery. JAMA. 2016;315(2):150. doi: https://doi.org/10.1001/jama.2015.18118
8. Mamplekou E, Komesidou V, Bissias C, Papakonstantinou A, Melissas J. Psychological Condition and Quality of Life in Patients with Morbid Obesity Before and After Surgical Weight Loss. Obes Surg. 2005;15(8):1177-1184. doi: https://doi.org/10.1381/0960892055002356

9. Livhits M, Mercado C, Yermilov I, et al. Preoperative Predictors of Weight Loss Following Bariatric Surgery: Systematic Review. Obes Surg. 2012;22(1):70-89. doi: https://doi.org/10.1007/s11695-011-0472-4

10. Тарабрина Н.В. Практикум по психологии посттравматического стресса. - СПб: Питер, 2001. - C. 146-157. [Tarabrina N.V. Workshop on the psychology of post-traumatic stress. - St. Petersburg: Peter, 2001. - P. 146-157. (In Russ).].

11. R Core Team (2018). R: A language and environment for statistical computing. R Foundation for Statistical Computing, Vienna, Austria. URL https://www.R-project.org/

12. Petroni ML, Villanova N, Avagnina S, et al. Psychological Distress in Morbid Obesity in Relation to Weight History. Obes Surg. 2007;17(3):391-399. doi: https://doi.org/10.1007/s1 1695-007-9069-3

\section{ИНФОРМАЦИЯ ОБ АВТОРАХ [AUTHORS INFO]:}

*Неймарк Александр Евгеньевич, к.м.н. [Aleksandr E. Neimark, MD, PhD]; адрес: Россия, 197341, Санкт-Петербург, ул. Аккуратова, д. 2 [address: 2 Akkuratov street, 197341 St. Petersburg, Russia]; ORCID: http://orcid.org/0000-0003-4925-0126; eLibrary SPIN: 6554-3217; e-mail: sas_spb@mail.ru

Еганян Шушанна Арамовна, к.психол.н. [Shushanna A. Eganian, PhD in psychology];

ORCID: https://orcid.org/0000-0002-5982-7884; eLibrarySPIN: 6961-9592; e-mail: shushanna25@mail.ru Гальченко Максим Иванович, старший преподаватель [Maxim I. Galchenko, seniorlecturer]; ORCID: https://orcid.org/0000-0002-5476-6058; eLibrarySPIN: 8858-2916; e-mail: maxim.galchenko@gmail.com

*Авторы, ответственные за переписку.

\section{ЦИТИРОВАТЬ:}

Неймарк А.Е., Еганян Ш.А., Гальченко М.И. Психологические факторы в снижении избыточной массы тела после бариатрических операций // Ожирение и метаболизм. - 2019. - Т.16. — №3. - C.62-68. doi: https://doi.org/10.14341/omet10043

\section{TO CITE THIS ARTICLE:}

Neimark AE, Eganian SA, Galchenko MI. Psychological factors in reducing excess body weight after bariatric surgery. Obesity and metabolism. 2019;16(3):62-68. doi: https://doi.org/10.14341/omet10043 